\title{
Surgical oncology of the head and neck district during COVID-19 pandemic
}

\author{
Giovanni Salzano $^{1} \cdot$ Fabio Maglitto $^{1} \cdot$ Agostino Guida $^{1}$ (1) $\cdot$ Francesco Perri ${ }^{2} \cdot$ Maria Grazia Maglione ${ }^{1}$.

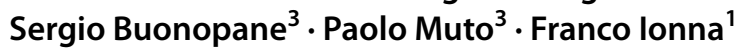

Received: 30 June 2020 / Accepted: 23 November 2020 / Published online: 4 January 2021

○) Springer-Verlag GmbH Germany, part of Springer Nature 2021

\begin{abstract}
Purpose A new member of the Coronaviridae family caused a worldwide pandemic emergency called Coronavirus disease 2019 (COVID-19). Health care workers who come into contact with the upper aero-digestive tract during diagnostic and therapeutic procedures, such as otolaryngologists, oral and maxillofacial surgeons, and head and neck surgeons, may undergo profound changes in their activities and are particularly at risk. We analysed the impact of COVID-19 on our oncological surgical activity.

Methods To address the emergency and guarantee safety of patients referred to our Unit, reproducible guidelines were followed. Surgical activity data during COVID-19 were compared to previous years (2018 and 2019).

Results From 21st February to 25th of May 113 surgical procedures were performed. The average of the two selected years (2018-2019) is 84.5 , showing an increase of $34.5 \%$ of our activities (statistically significant, $p=0.0011$ ). No patient showed perioperative or postoperative contagion.

Conclusion Due to the conversion of regular Hospitals into COVID Centers, Cancer Centers may encounter an increased demand for procedures. Following strict guidelines, it seems possible to face surgical activity on cancer patients and respect standard procedures aimed at containing the spread of COVID-19 infection.
\end{abstract}

Keywords COVID-19 $\cdot$ Head and neck cancer $\cdot$ Surgical oncology $\cdot$ ENT

\section{Introduction}

From December 2019, a new member of Coronaviridae family, the Severe Acute Respiratory Syndrome Coronavirus-2 (SARS-CoV-2) caused a pandemic called Coronavirus disease 2019 (COVID-19). COVID-19 mainly strikes the respiratory tract, ranging from mild respiratory symptoms to severe viral pneumonia with respiratory failure and death [1].

Agostino Guida

a.guida@istitutotumori.na.it

1 Division of Maxillo-Facial and ENT Oncological Surgery, Istituto Nazionale Tumori, IRCCS Fondazione G. Pascale, Via M. Semmola 52, 80131 Naples, Italy

2 Head and Neck Medical and Experimental Oncology, Istituto Nazionale Tumori, IRCCS Fondazione G. Pascale, Naples, Italy

3 Radiation Oncology Unit, Istituto Nazionale Tumori, IRCCS Fondazione G. Pascale, Naples, Italy
The main pathway of contagion is through respiratory droplets from symptomatic patients [1]. Asymptomatic patients may be contagious too, but, according to WHO, the contagion of COVID-19 is primarily occurring from people when they have symptoms or just before they develop symptoms, thus more research is needed in this field, as it is not clear to what extent asymptomatic patients may pass the virus [2].

Epithelial cells of the oral mucosa are rich in ACE2receptors, which represent the proteins through which SARS-CoV-2 penetrates in host cells. This feature may explain the high concentration of viral particles detected in the oral cavity of infected patients, thus favouring infection through sol [1].

For these reasons, health care workers who come into contact with the upper aero-digestive tract during diagnostic/ therapeutic procedures in patients with suspected/confirmed COVID-19, such as oral and maxillofacial surgeons, otolaryngologists and head and neck surgeons, are, particularly at risk [3]. 
Right precautions are mandatory. FFP3-N95 masks are not sufficient to control the dissemination of the disease between the aforementioned categories of healthcare professionals taking care of at-risk patients [4-7]. Indeed, it is not until Powered Air Respiratory Protection (PAPR) has been introduced that the transmission of the SARS-CoV-2 was controlled among medical workers [8].

In Italy, COVID-19 spread has been very peculiar [9]: up until July 2020, the 5 most-affected regions accounted for more than $75 \%$ of cases (Lombardia 39.1\%; Piemonte 13\%; Emilia Romagna 11.9\%; Veneto 8\%; Liguria 4.2\%; total $76.2 \%$ ), all of them being in northern Italy. Our region, Campania, which is in the southern Italy, accounted for $2 \%$ cases (total 4762 cases), unequally distributed among the different cities (Avellino: 561 cases; Benevento: 218; Caserta: 588; Naples: 2693; Salerno: 702). More than half Campania cases thus occurred in Naples, the most populated area of the region. Despite not being high when compared to the rest of our Country/other European regions, such numbers resulted in a conversion of 56 Campania hospitals -almost the whole hospital network- into specialized centers for the treatment of COVID-19 (COVID Centers, CCs) [10]. Almost every hospital thus blocked/reduced most of their activity during the COVID-19 peak period, including cancer care, and this resulted in a conveying of oncological patients to our hospital, which is the only Cancer Center in our region.

Consequentially, due to all these factors, during this period head and neck malignancies management represents a particularly complicated challenge to be faced by head and neck surgeons. To our knowledge, universally accepted protocols for head and neck inpatient/outpatient procedures during COVID-19 do not exist. To address the emergency and to guarantee the safety of patients referring to our Unit (Department of Maxillofacial and Ear-Nose-Throat Oncological Surgery of the National Cancer Institute of Naples "G. Pascale", Italy), reproducible guidelines have been crafted and followed. We thus analysed our activity, also comparing it to previous years, in order to understand the possible influence of the COVID pandemic on the outcomes of head and neck cancer surgery.

\section{Materials and methods}

We followed our Institute's COVID-19 guidelines for infection control and then added specific measures for peculiar head and neck outpatient/inpatient activity. General measures include: distribution of personal protective equipment (PPE: FFPP2/3 mask, disposable gown, face mask/shield), training on infection prevention measures, triage, controlled affluence, reorganization of wards, more frequent cleaning/ventilation of the environment, distribution of surgical masks to every patient. Clear protocols were established with the Anaesthesia Department, nursing staff, Recovery Unit personnel, and Infection Control personnel. The general policy was to try to be free from COVID-19 patients, to ensure that enough clinical and intensive-care capacity can be reserved for critical cancer surgical procedures or management of side effects of systemic anticancer treatment. Infected/suspected patients were quickly transferred to CC for further investigation/care [11].

\section{Precautions for outpatient activities}

We scheduled appointments for outpatient clinical examination in such a way as to limit the number of patients waiting to be examined, to apply social distancing and avoiding contact among patients during their way in/out. PPE is administered to physicians/nurses during every outpatient procedure which exposes the operator to respiratory droplet/contact with patients not wearing masks: surgical procedures, mouth inspection, ENT fibroscopy. About the latter, specific measures have been codified for nose/pharynx/larynx fibroscopy. A flexible fiberscope with the smallest possible diameter was preferred, in order to reduce nausea/cough and possible traumatic iatrogenic bleeding, with a disposable device covering the endoscope. Furthermore, to reduce patients' nausea/ cough, anaesthetic was used in every patient, preferring gel to spray [12], to reduce aerosol production.

\section{Precautions for inpatient activities}

Prior to inpatient hospitalization, every patient undergoes a serological test for SARS-CoV-2 antibodies. Patients with positive antibodies are quickly transferred to $\mathrm{CCs}$ for further investigation/care. Particular attention and adequate protection are due during the transfer of SARS-CoV-2-positive/ doubtful patients. Once a patient is admitted to the ward, PPE is administered to physicians/nurses during every inpatient procedure which exposes the operator to respiratory droplet/contact with patients not wearing masks: surgical procedures, mouth inspection, ENT fibroscopy (as described before), post-surgical dressing.

\section{Quantitative and qualitative analysis}

Qualitative and quantitative data of our surgical procedures have been performed, in order to understand if our activity had undergone any sensitive changes compared to previous years (2018-2019). Results were then evaluated at the T-student ( $p$-value) statistical test with a 95\% confidence interval (CI 95\%, $p<0.05)$ to essay the statistical significance of a possible variation. Complications and patients/ personnel contagion have been examined and analysed as descriptive data. 


\section{Results}

In our Department, during the Covid-19 outbreak, ranging from 21 February to 25 May 2020, 113 patients have undergone general anaesthesia inpatient surgical procedures for head-neck malignancies (mean: 37.6 interventions/month). Surgical procedures have been performed following the aforementioned COVID-19 protocols.

\section{Surgical activity}

Benign tumour surgery has been suspended; on the other hand, there has been an increase in malignant neoplasm procedures. Trans Oral robotic surgery (TORS) was not suspended, also because, as revealed in literature, for selected cases, this approach could reduce hospitalization times [13]. Table 1 summarizes the procedures performed. "Biopsy" category refers to single neck node harvesting for diagnosis/characterization of suspected haematological disease and incisional biopsies requiring general anaesthesia (e.g.: nasopharynx); after biopsy and subsequent diagnosis are performed, patients undergo treatment as per guidelines. The lowest number of surgical procedures is represented by thyroid cancers $(n=3)$. These cases were referred from our Thyroid Surgery Unit, as they needed special neck metastasis management (e.g.: carotid involvement). Resident thyroid surgeons co-operated with us during the procedure. On the other hand, among the most frequent procedures, parotid cancer represented a high percentage of our activity $(16 \%)$. We reckon that such high numbers of parotid procedures are due to the shortage of other referral centers in our region, as suggested by the fact that 2020 numbers are

Table 1 Surgical procedures during COVID-19 pandemic performed at Department of Maxillofacial Surgery and ENT of the National Cancer Institute of Naples "G. Pascale", Italy (From 25th February 2020 to 21st May 2020)

\begin{tabular}{ll}
\hline Surgical procedures & Number of procedures \\
\hline Biopsies (95\% Neck Lymph nodes) & $19(16.8 \%)$ \\
Parotidectomy & $18(16 \%)$ \\
T1,T2 larynx cancers & $15(13.2 \%)$ \\
Removal of oral cavity tumors & $14(12.4 \%)$ \\
Neck dissections & $9(7.9 \%)$ \\
FESS & $7(6.2 \%)$ \\
Minor surgical procedures (surgical hemo- & $8(7.1 \%)$ \\
stasis, fistula closure etc....) & \\
Laryngectomies & $5(4.4 \%)$ \\
TORS & $6(5.4 \%)$ \\
Thyroidectomies & $3(2.6 \%)$ \\
Electrochemotherapies & $4(3.6 \%)$ \\
Tracheotomies & $5(4.4 \%)$ \\
\hline
\end{tabular}

so far even higher than data from 2019 (41 parotid over 393 total procedures, $10 \%)$ and $2018(50 / 363,13 \%)$ activity. We then compared our February-May 2020 activity to the same period in 2018 and 2019. Analysing our data, 101 surgical procedures were performed during the same period in 2019 and 68 in 2018. The average of the two selected years is 84.5 procedures. In 2020, we thus achieved an increase of 34.5\% of our surgical activities (Table 2). We calculated that if a mean of 37.6 intervention/month is confirmed over the rest of 2020, we will have a statistically significant increase in our surgical annual activity if compared to the 2-year period 2018-2019 ( $p=0.0011)$. Interestingly, if we compare our surgical annual activity between 2018 (363 surgical procedures, mean 30.2/month) and 2019 (393 surgical procedures, mean 32.8 /month) as control, we do not have a statistically significant difference $(p=0.3)$.

\section{Complications and contagions}

No complications related to COVID-19 were recognized postoperatively. Patients showed surgical-related complications, such as haematoma ( $n=6,2$ after parotid surgery, 2 laryngectomies, 1 neck dissection, 1 lymph node biopsy), haemorrhage ( $n=2,1$ FESS, 1 neck dissection), surgical wound infection ( $n=1$, laryngectomy), transient nerve weakness ( $n=4,3$ parotid surgery, 1 thyroid surgery). Haemorrhages were successfully controlled through surgical haemostasis; infection was controlled with aimed antibiotic therapy after swabbing for microbiological analysis. Six patients needed Intensive Care Unit (ICU) after surgery for controlled awakening (5 laryngectomies, 1 neck dissection).

No patients developed COVID-19 infection/symptoms during hospitalization; they were also interviewed during the first postoperative clinical exam (at 7-10 days) and no one developed symptoms after discharge.

Table 2 Number of surgical procedures during the COVID-19 pandemic and during the 2 previous years and their statistical analysis through Student's $t$-test (CI 95\%, $p<0.05 ; 2020$ data has been obtained projecting COVID-19 lockout monthly mean of procedures for 12 months)

\begin{tabular}{llll}
\hline Month & 2018 & 2019 & 2020 \\
\hline March & 23 & 44 & 45 \\
April & 27 & 35 & 37 \\
May & 18 & 22 & 36 \\
Total & 68 & 101 & 113 \\
Total per whole yr & 363 & 394 & 451 (projection) \\
Monthly mean & 30.25 & 32.8 & 37.6 \\
2018 vs. 2019 & $\begin{array}{l}p=0.3 \text { (not } \\
\text { signifi- }\end{array}$ & 2020 (projec- & $\begin{array}{c}\text { tion) vs. } \\
\text { cant) }\end{array}$ \\
& 2018/19 & (projection, \\
& significant) \\
\hline
\end{tabular}




\section{Discussion}

During this pandemic, cancer patients' management becomes more challenging, as they represent a high-risk group. Cancer patients are generally more vulnerable to infections due to their underlying disease and typically immunosuppressed state, which implies a greater risk of serious viral complications which may result in the need for intensive care or even death [14].

The media reported frequent reduction of cancer treatments to prioritize COVID-19 patients [15]. Despite Campania's, our region, contagion number not being high when compared to the rest of Italy, conversion of 56 Campania hospitals into CC [10] was needed to face the emergency. Such hospitals blocked most of the rest of their activity, including cancer care. As we have shown, we recorded a statistically significant increase in our oncological surgical activity when compared to previous years. We believe that this increase is due to the fact that many surrounding hospitals, normally performing head and neck oncology, have suspended this activity once converted into CC. Conversion of Hospitals into CC may have thus increased the number of patients referred to our Institute. Consequentially, during this period, head and neck malignancies management represents a particularly complicated challenge to be faced by head and neck surgeons. Malignancies of the head and neck district can produce serious respiratory difficulties when left untreated $[16,17]$ and thus postponing surgical therapy of such neoplasms may put patients' life in great danger. The great effort needs to be put into granting continuity of care, even in such difficult times.

This concerns especially patients affected by advanced laryngeal carcinomas (stages III or IV)—which represent approximately $60 \%$ of all laryngeal cancers referred to our Unit. Surgery of laryngeal cancer should not be postponed, as patients commonly experience hoarseness, dysphonia, swallowing and severe dyspnoea, which may result in emergency tracheotomy [18]. Being dyspnoea a common symptom in COVID-19, these patients need particular protection from contagion, but delayed therapies should be avoided, to guarantee the highest survival rate.

No COVID-related complications were recorded in our Department during the examined period. Possibilities of increased surgical-related complications during COVID19 have been studied by many Authors. A multicentre cohort study finds that COVID-19 positive patients have an increased risk of complications for hip surgery [19], but another study highlights that this difference is minimal $(5 \%)$ in delicate procedures as emergency general surgery [20]. Still, our data seems to be in accordance with the literature as, with an efficient screening procedure, Head and
Neck elective oncological surgery is generally considered a safe activity [21]. Although chemo- and radio-therapy treatments are options for selected types of head and neck malignancies, surgery must be preferred when guidelines indicate it as primary choice [22], even in times like these when surgical treatment exposes operators to greater risk of infection; using correct protective measures is necessary to contain contagion [23, 24].

The social impact of Head and Neck oncology is well documented in the scientific literature. Treatments are essentially surgical and radical, resulting in high stress/ poor quality of life for patients and their families. It has been shown that patients frequently suffer from high levels of emotional distress, resulting in an elevated risk of suicide [25] higher than in those with cancers in other sites. The impossibility of caring for Head and Neck patients -which are mainly treated with surgery- may result in profound consequences, thus therapies need to continue unaltered even during these difficult times. Surgery Units involved in the therapy of Head and Neck neoplasms need to be prepared in order not to reduce their activity.

\section{Conclusion}

Our study shows that Head and Neck Oncological Surgery Units need to be prepared in order not to reduce their activity or in some cases to face an increase of procedures, as many Hospitals are converted into CC. Despite difficulties that can be encountered in this period, with due precautions/regulations we showed that surgical activity can be carried out, even facing an increase of activity for Cancer Centers. Our Unit has tried not to reduce therapies of cancer patients and at the same time respect the procedures aimed to contain the COVID-19 infection. Such preventive procedures seem effective, not slowing down inpatient surgical activity nor impacting an increased demand for procedures.

Funding None.

\section{Compliance with ethical standard}

Conflict of interest The authors declare that they have no conflict of interest.

Ethical approval Not applicable.

Research involving human/animals participants No research involved human/animals was performed.

Informed consent Not applicable. 


\section{References}

1. Xu H, Zhong L, Deng J et al (2020) High expression of ACE2 receptor of 2019-nCoV on the epithelial cells of oral mucosa. Int J Oral Sci 12(1):8

2. Transmission of SARS-CoV-2: implications for infection prevention precautions in World Health Organization Website. https:// www.who.int/news-room/commentaries/detail/transmission-ofsars-cov-2-implications-for-infection-prevention-precautions

3. Givi B, Schiff BA, Chinn SB et al (2020) Safety recommendations for evaluation and surgery of the head and neck during the COVID-19 pandemic. JAMA Otolaryngol Head Neck Surg 146(6):579-584

4. Howard BE (2020) High-Risk Aerosol-generating procedures in COVID-19: respiratory protective equipment considerations. Otolaryngol Head Neck Surg 163(1):98-103

5. Patel ZM, Fernandez-Miranda J, Hwang PH et al (2020) Letter: precautions for endoscopic transnasal skull base surgery during the COVID-19 pandemic. Neurosurgery 87(1):E66-E67

6. Chee VWT, Khoo MLC, Lee SF et al (2004) Infection control measures for operative procedures in severe acute respiratory syndrome-related patients. Anesthesiology 100(6):1394-1398

7. Centers for Disease Control and Prevention. https://www.cdc.gov/ sars/clinical/respirators.html

8. Dan L, Haiyang W, Rong Y et al (2020) Integrated infection control strategy to minimize nosocomial infection of coronavirus disease 2019 among ENT healthcare workers. J Hosp Infect 104(4):454-455

9. Characteristics of SARS-CoV-2 patients dying in Italy. Report based on available data on July 22nd, 2020. https://www.iss.it/ web/iss-en

10. DECRETO-LEGGE 19 maggio 2020, n. 34 Misure urgenti in materia di salute, sostegno al lavoro e all'economia, nonche' di politiche sociali connesse all'emergenza epidemiologica da COVID-19. https://www.gazzettaufficiale.it/eli/ id/2020/05/19/20G00052/sg

11. Mansoor SN, Gill ZA, Rathore FA et al (2020) Establishing and managing a quarantine and isolation center in COVID-19 pandemic. J Pak Med Assoc 70(5):S11-S14

12. ENTUK.Guidance for ENT during the COVID-19 pandemic (2020) https://www.entuk.org/guidance-ent-during-covid-19pandemic

13. Thomas WW, Brant J, Chen J et al (2018) Clinical factors associated with reoperation and prologed length of stay in free tissue transfert to head and neck defects. JAMA Facial PlastSurg $20: 154-159$
14. Perri F, Ionna F, Longo F et al (2020) Immune response against head and neck cancer: biological mechanisms and implication on therapy. TranslOncol 13(2):262-274

15 Ralli M, Greco A, de Vincentiis M (2020) The Effects of the COVID-19/SARS-CoV-2 pandemic outbreak on otolaryngology activity in Italy. Ear Nose Throat J. https://doi.org/10.1177/01455 61320923893

16. Liang W, Guan W, Chen R (2020) Cancer patients in SARSCoV-2 infection: a nationwide analysis in China. Lancet Oncol 21(3):335-337

17. Shedd DP, Carl A, Shedd C (1980) Problems of terminal head and neck cancer patients. Head Neck Surg 2(6):476-482

18. Steuer CE, El-Deiry M, Parks JR et al (2017) An update on larynx cancer. Cancer J Clin 67(1):31-50

19. Kayani B, Onochie E, Patil V (2020) The effects of COVID-19 on perioperative morbidity and mortality in patients with hip fractures. Bone Joint J 102:1-10

20. Seretis C, Archer L, Lalou L (2020) Minimal impact of COVID19 outbreak on the postoperative morbidity and mortality following emergency general surgery procedures: results from a 3-month observational period. Med Glas (Zenica). https://doi.org/10.17392 /1229-20

21. Brar S, Ofo E, Hyde $\mathrm{N}$ et al (2020) Outcomes of elective head and neck confirmed or suspected cancer surgery during the COVID-19 pandemic. Eur Arch Otorhinolaryngol. https://doi.org/10.1007/ s00405-020-06194-2

22. Vermorken JB, Mesia R, Rivera F (2008) Platinum-based chemotherapy plus cetuximab in head and neck cancer. N Engl J Med 359(11):1116-1127

23. Day AT, Sher DJ, Lee RC et al (2020) Head and neck oncology during the COVID-19 pandemic: reconsidering traditional treatment paradigms in light of new surgical and other multilevel risks. Oral Oncol 105:104684

24. De Felice F, Polimeni A, Tombolini V (2020) The impact of Coronavirus (COVID-19) on head and neck cancer patients' care. RadiotherOncol 147:84-85

25. Twigg JA, Anderson JM, Humphris G et al (2020) Best practice in reducing the suicide risk in head and neck cancer patients: a structured review. Br J Oral MaxillofacSurg S0266-4356(20):30314-30324

Publisher's Note Springer Nature remains neutral with regard to jurisdictional claims in published maps and institutional affiliations. 